\title{
Development of a Four-way Interface for Online Capillary Isoelectric Focusing-Electrospray-Mass Spectrometry (CIEF-ESI-MS)
}

\author{
Hai Dong Yu ${ }^{1,2}$, Byungjoo Kim ${ }^{1}$, Dae-Ho Shin ${ }^{3}$, and Seonghee Ahn ${ }^{1, *}$ \\ ${ }^{I}$ Division of Metrology for Quality of Life, Korea Research Institute of Standards and Science, Yuseong, Daejeon 305-600, Korea \\ ${ }^{2}$ Department of Bio-analytical Science, University of Science and Technology, Yuseong, Daejeon 305-333, Korea \\ ${ }^{3}$ Bio Medical Technologies Ltd., 21-10 Dongho-Ro 15-Gil, Jung-Gu, Seoul 100-894, Korea
}

Received December 11, 2013; Revised December 19, 2013; Accepted December 19, 2013

First published on the web December 30, 2013; DOI: 10.5478/MSL.2013.4.4.83

\begin{abstract}
A new interface for coupling CIEF and MS using a four-way cross has been developed in a single mechanical system. This new interface could be operated without the electric discontinuity and reinstallation of lines. Additionally, a bare fused silica capillary was facilitated as a spray needle to produce electrospray and to guide catholyte or sheath liquid. Focusing for CIEF was completed in a hanging droplet at the end of spray needle. This capillary spray needle also provided stable spray, enhanced the ionization efficiency and increased sensitivity. Results with carbonic anhydrase I showed that focusing and spraying were well completed with the new interface and the new spray needle.
\end{abstract}

Key words: CIEF-ESI-MS, Interface, Carbonic anhydrase I, Fourier transform mass spectrometry

\section{Introduction}

Conventional isoelectric focusing allows separating the amphoteric compounds such as peptides, proteins in slab gels according to their isoelectric point $(\mathrm{p} /){ }^{1}$ Capillary isoelectric focusing (CIEF) was first introduced by Hjerten et al. ${ }^{2}$ in 1985, which was performing isoelectric focusing within a fused silica capillary without slab gels. A mixture of carrier ampholytes and analytes is loaded into a separation capillary. Separation is based on the focusing of analytes and carrier ampholytes at locations of inherent isoelectric points within a $\mathrm{pH}$ gradient across the capillary under high voltage. There are three discrete steps for performing CIEF, loading, focusing and mobilization. Sample mixed with carrier ampholytes is loaded through the whole column from the inlet to the outlet. After sample loading, focusing proceeds with applying high voltage across the capillary. The $\mathrm{pH}$ gradient forms by solutes migration to the appropriate position along the gradient and analytes are separated by their $p$ I values. After the focusing was completed, it

\section{Open Access}

*Reprint requests to Dr. Seonghee Ahn

E-mail: e-mail: sahn@kriss.re.kr

All MS Letters content is Open Access, meaning it is accessible online to everyone, without fee and authors' permission. All MS Letters content is published and distributed under the terms of the Creative Commons Attribution License (http://creativecommons.org/licenses/by/3.0/). Under this license, authors reserve the copyright for their content; however, they permit anyone to unrestrictedly use, distribute, and reproduce the content in any medium as far as the original authors and source are cited. For any reuse, redistribution, or reproduction of a work, users must clarify the license terms under which the work was produced. is necessary to mobilize or elute the contents of the capillary past a detector installed at the end of capillary (outlet bounce). CIEF is an alternative to the traditional slab gel electrophoresis because it allows fast and automated analyses with high resolution and requires small sample amount.

Coupling CIEF with mass spectrometry (MS) by electrospray ionization was first demonstrated by Tang et al. ${ }^{3}$ The CIEF-ESIMS has been more powerful techniques providing information for protein structures and characterization based on their $p \mathrm{I}$ and molecular weights, ${ }^{3-8}$ which is analogous to the conventional two-dimensional (2D) polyacrylamide gel electrophoresis (PAGE). However, in spite of its potential advantages and applications for protein analysis, online CIEF-MS coupling using ESI interfaces presents several technical difficulties. First, most of the traditional CIEF reagents are not ESI compatible, such as strong inorganic acids and bases which are usually used for catholyte and anolyte. They can be replaced by volatile organic acids and bases for ESI-MS. Another big challenge was the carrier ampholytes that were coeluted with analytes, as they would suppress ionization of analytes and create spectral interferences. Coaxial sheath flow was introduced to interfacing capillary electrophoresis mass spectrometry (CE/MS) to enhance the performance of spray and reduce many of CE/MS limitations. ${ }^{9,10}$ The coaxial sheath flow (1-5 $\left.\mu \mathrm{L} / \mathrm{min}\right)$ of liquid establishes the electrical contact with the $\mathrm{CE}$ effluent to the cathode of $\mathrm{CE}$, which also provides electrospray voltage. This coaxial sheath flow was also applied to the electrospray process for CIEF-ESI-MS interfacing by Tang and co-workers using the Finnigan MAT electrospray kit. ${ }^{3}$ In this case, the CIEF capillary inlet end was put into the anolyte reservoir and the outlet end which was mounted within the electrospray probe was put into 
the catholyte reservoir during focusing. Once the focusing was completed, the electric potential was turned off and the outlet reservoir was removed, and the outlet end was reinstalled into the electrospray needle. During the mobilization step, the sheath liquid flow is delivered by liquid sheath tubes and two HV power supplies were used for delivering the electric potentials at the inlet electrode and at the electrospray needle. Clake and Naylor developed another strategy producing a static 'hanging' drop of catholyte at the tip of spray needle for focusing. ${ }^{6}$ In the focusing stage, the capillary column, filled with sample and ampholyte solution, was installed to pass through the electrospray needle (sheath liquid line) with its tip a few milimeter outside the electrospray needle. Catholyte was delivered co-axially through the sheath liquid line enabling the formation of a static 'hanging' drop of catholyte at the tip of capillary column. During focusing, the hanging drop works as the catholyte reservoir for the CIEF. Once focusing was completed, the electrical potential was turned off and the sheath liquid line was flushed with sheath liquid solution (to be used for ESI). The end of capillary column was then repositioned to a distance of $0.5 \mathrm{~mm}$ out of the spray needle. During analyte mobilization, the sheath liquid solution was co-axially delivered. Kuroda et al. installed the end of capillary through a modified stainless steel tubing in the laboratory-built CE system and was positioned $c a$. $0.5 \mathrm{~mm}$ outside the outlet of the metal tubing. ${ }^{7}$ The coaxial sheath liquid was administered at a flow rate of $2 \mu \mathrm{L} / \mathrm{min}$ through the metal tubing. These previous methods for interfacing CIEF and MS were preceded with electric discontinuity and reinstallation of the spray needle. Even in those cases, a line delivering catholyte liquid should be mechanically exchanged to a line for delivering sheath liquid.

In this study, a new interface for CIEF and MS was developed using a four-way cross to deliver the catholyte and the sheath liquid within a single mechanical system. Four-way cross system does not need to stop the electricity neither to move the spray tip when starting the operation of CIEF/MS. In addition, the bare fused silica capillary was employed as a spray needle to produce stable electrospray as well as to guide the sheath liquid. The result in loading carbonic anhydrase I (CA I) onto the CIEF capillary column showed this new four-way cross interface and the new spray needle operated well providing a peak in electropherogram. Coupling with CIEF and FTMS showed a charge and isotopic distribution in mass spectrum.

\section{Experimental Section}

\section{Materials}

Ammonium hydroxide and acetic acid were purchased from Sigma (St. Louis, MO, USA). Pharmalyte ( $\mathrm{pH} 3-10$; GE Healthcare) was used as a carrier ampholyte to generate a $\mathrm{pH}$ gradient at $25 \mathrm{kV}$. Samples were desalted using Millipore Amicon Ultra- $0.5 \mathrm{~mL}$ centrifugal filter and premixed with the carrier ampholyte (final concentration of $1 \%, v / v$ ). Then samples were centrifuged at $12000 \mathrm{rpm}$ for $10 \mathrm{~min}$ and subjected to sonication to remove gas bubbles.

\section{CIEF-ESI-MS}

The column length was $100 \mathrm{~cm}(70 \mathrm{~cm}$ effective length for CIEF-UV). Ammonium hydroxide ( 1\%, v/v, $\mathrm{pH} \sim 9.5)$ solution was used as the catholyte and acetic acid $(\sim 1 \%, \mathrm{v} /$ $\mathrm{v}, \mathrm{pH} \sim 2.6)$ solution was used as the anolyte. The entire column was filled with the sample which was prepared with $1 \%$ ampholyte. CIEF experiments were performed using PRINCE Crystal $650 \mathrm{CE}$ system (Emmen, Netherlands) and UV detection (Lambda 1010 UV-Vis; Bischoff, Germany) was carried out at $280 \mathrm{~nm}$. A constant voltage of $25 \mathrm{kV}$ was applied during focusing $(20 \mathrm{~min})$ and hydrodynamic mobilization ( $\sim 25$ mbar). Mass spectra were acquired by $12 \mathrm{~T}$ Fourier Transform Mass Spectrometer (FTMS, Agilent, Lake Forest, CA, USA) in positive mode with $1.6 \mathrm{~s}$ scan rate. Mass range of FTMS was performed in $\mathrm{m} / \mathrm{z}$ 600 2000. Sheath liquid solution consisted of $40 \%$ methanol, $58 \%$ water and $2 \%$ acetic acid $(\mathrm{v} / \mathrm{v} / \mathrm{v})$ and delivered at a flow rate of $2 \mu \mathrm{L} / \mathrm{min}$ by a syringe pump. $3.5 \mathrm{kV}$ was applied to the four-way cross for ESI spraying which was monitored by a CCTV camera to check the shape and stability of ESI.

\section{Preparation of Coated Column}

Hydroxypropyl cellulose (HPC) powder was dissolved in water at room temperature to a final concentration of $5 \%$ (w/w). The polymer solution was left overnight to eliminate bubbles. The capillary column was filled with the HPC solution by a syringe pump, and the excess solution was drained out by using $\mathrm{N}_{2}$ gas at $30 \mathrm{psi}$. The polymer layer coated on the capillary inner wall was immobilized by heating from $60{ }^{\circ} \mathrm{C}$ to $140{ }^{\circ} \mathrm{C}$ at $5{ }^{\circ} \mathrm{C} / \mathrm{min}$ and then keeping at $140^{\circ} \mathrm{C}$ for $20 \mathrm{~min}$ in a gas chromatographic oven with $\mathrm{N}_{2}$ gas at 30 psi.

\section{Results and Discussion}

Figure 1(a) demonstrates a diagram of the four-way interface for CIEF-ESI-MS. The new interface employed the four-way cross for delivering the catholyte and the sheath liquid separately. A CIEF capillary column was inserted from one port of the four-way cross and passed through the spray needle (sheath liquid line) installed at the opposite port of the four-way cross. The capillary column was fixed to protrude $c a .1 \mathrm{~mm}$ outside of the spray needle. The other two ports of the four-way cross were connected to lines delivering catholyte and sheath liquid separately. The catholyte or sheath liquid was delivered at a flow rate of $2 \mu \mathrm{L} / \mathrm{min}$ to the center of the four-way cross and flowed through the spray needle (inside the outer tube of CIEF capillary). For performing CIEF separation, the capillary column was filled with sample solution from the other end of the column (anode side). During focusing, catholyte was co-axially delivered to the end of spray needle for the formation of a "hanging drop" and the end of capillary column was soaked into the "hanging drop" (Figure 1(b)). 

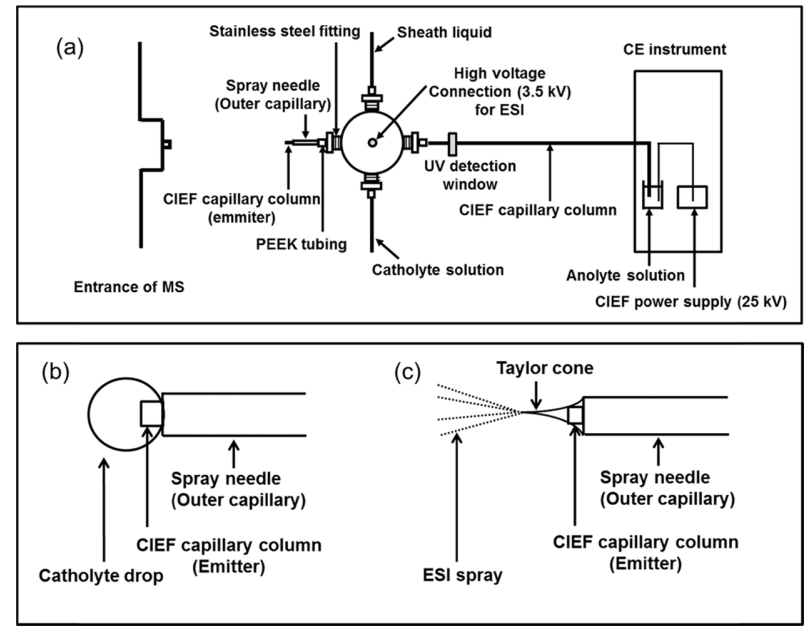

Figure 1. A schematic diagram of four-way interfacing for online CIEF-ESI-MS (a). A CIEF capillary column was placed through the four-way cross and extruded $1 \mathrm{~mm}$ from the spray needle which is bare fused silica capillary. The other ways of the cross were connected to sheath liquid and catholyte solution, respectively. Catholyte solution was delivered to the end of column and produced the "hanging-drop" catholyte during focusing step (b). The emitter of CIEF capillary column was soaked inside of the catholyte drop. After focusing was completed, the catholyte solution was stopped and the sheath liquid was delivered to the end of capillary column with applying $3.5 \mathrm{kV}$ for ESI spray during mobilization step (c).

The electric circuit for focusing was completed by applying high positive voltage $(25 \mathrm{kV})$ at the anode side and grounding the cathode side (the body of four-way cross which is connected to the hanging drop by sheath liquid inside spray needle). In this experiment, a catholyte 'hanging drop' forms and drops away for every 6 minutes when the catholyte flow kept at $2 \mu \mathrm{L} / \mathrm{min}$. Once focusing was completed, the mobilization step started with starting sheath liquid flow and stopping catholyte flow. In this way, liquid co-axially flowing through the spray needle was switched from catholyte to sheath liquid without manual exchanging of lines and electrical discontinuity. Then, $3.5 \mathrm{kV}$ applied to the cross for producing ESI spray (Figure 1 (c)) with maintaining $25 \mathrm{kV}$ at the anode side for keeping the $p$ I gradient. Switching volume from catholyte to sheath liquid, which is composed of the volume from the center of the cross to the spray needle, was estimated to be $19 \mathrm{~nL}$. The sheath liquid line can be switched from catholyte to sheath liquid within a minute. Therefore, in this design, CIEF focusing stage can be easily switched to the electrospray formation for MS detection of CIEF eluent without any mechanical changes of lines.

In the early attempt for interfacing CIEF-MS, a commercial electrospray adapter kit or a modified metal tubing spray needle was employed to apply the high voltage for electrospraying. ${ }^{3,4,7}$ However, these metal spray needles
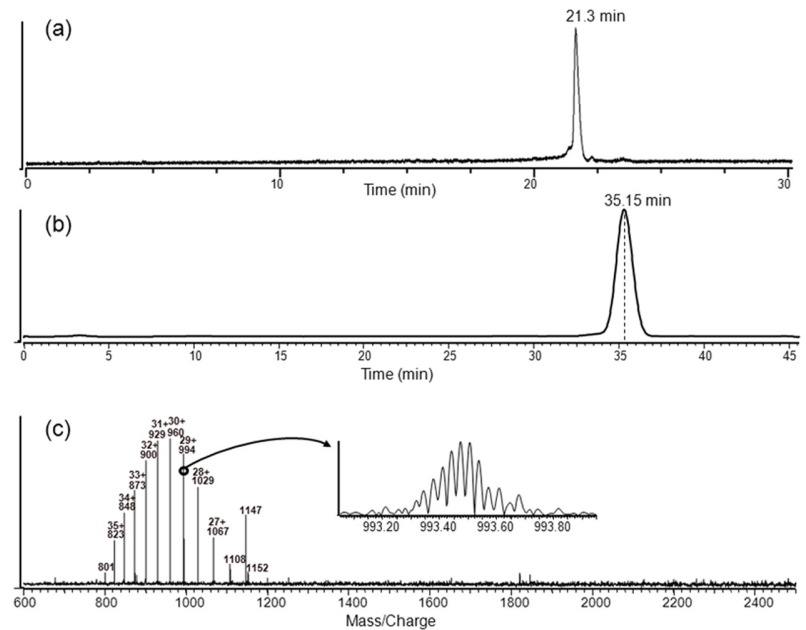

Figure 2. A typical electropherogram of CA I ( $p$ I 6.6, $0.1 \mathrm{mg}$ / $\mathrm{mL}$ ) acquired by CIEF-UV (a). A reconstructed electropherogram of CA I was acquired by four-way interfacing online CIEF-ESI-FTMS (b). The difference of migration time for CIEF-ESI-FTMS from that for CIEF-UV was due to the difference of effective length of analytical capillary column. The MS scan was accomplished over the $\mathrm{m} / \mathrm{z}$ range $600-2500$ with scan rate $1.6 \mathrm{~s}$. The MS scan spectrum at retention time $35.15 \mathrm{~min}$ (c) showing the charge distribution of CA I from +36 to +26 . The enlarged MS spectrum showed the isotopic distribution of charge state of $[\mathrm{M}+29 \mathrm{H}]^{29+}(\mathrm{m} / z$ 994) indicating the resolution of FTMS.

had limitation to provide a stable spray with high voltage causing electric arching between the spray needle and the entrance for mass spectrometry in CIEF-MS system. In this study, a bare fused silica capillary was used as a spray needle (outer capillary). The inner dimension of the capillary spray needle (I.D. $530 \mu \mathrm{m}$ ) was larger than the outer dimension of CIEF capillary column (O.D. $360 \mu \mathrm{m})$, therefore the CIEF capillary column pass through and protrude beyond it at the ESI side. This double capillary needle system provided stable spray, enhanced the ionization efficiency and increased sensitivity.

Figure 2(a) shows a typical electropherogram of carbonic anhydrase I (CA I: $p$ I 6.6, MW 28784) obtained by CIEFUV with typical setting and (b) shows a total ion electropherogram by CIEF-ESI-MS with newly designed four-way interface. An analytical capillary column (I.D. $50 \mu \mathrm{m}$, O.D. $360 \mu \mathrm{m}$ ) for CIEF separation was coated with $5 \%$ hydroxypropyl cellulose (HPC) solution twice and the method $^{11}$ to coat a column is described in experimental section. Figure 2(b) proved that the new interface provides stable electrospray with overcoming limitations observed with previous systems using the metal tubing spray needle. Difference of migration time for CA I at $22.1 \mathrm{~min}$ by CIEFUV in Figure 2(a) and at $35.2 \mathrm{~min}$ by CIEF-ESI-MS in Figure 2(b) is due to the difference of effective length for CIEF-UV, as the detector was located at $30 \mathrm{~cm}$ from the 
end of the CIEF capillary. The hanging-drop focusing with four-way cross was well accomplished showing a peak in Figure 2(b). Figure 2(c) is the mass spectrum at $35.2 \mathrm{~min}$ showing charge state distribution from $+36(\mathrm{~m} / \mathrm{z} 801)$ to $+26(\mathrm{~m} / \mathrm{z} 1108)$ of CA I ( $/ \mathrm{z} / 1147$ is an instrument noise) . One of the charge state $(+29, \mathrm{~m} / z$ 994) was enlarged in the circle of Figure 2(c) showing the isotopic mass distribution by FT/MS. It is noticed that the peaks for CA I on the electropherogram by CIEF-ESI-MS is somewhat wider than the peaks on the electropherogram by typical CIEFUV. Operational parameters, for examples rate of sheath flow, position of the tip of CIEF capillary at the ESI side, were under optimization to improve the separation resolution.

\section{Conclusions}

In summary, the new interface for online CIEF-ESI-MS was developed using the four-way cross. The fused silica capillary outer tubing facilitated the focusing and stable electrospray transferring catholyte or sheath liquid. The catholyte hanging-drop at the end of capillary column leaded the complete focusing shown on the reconstructed electropherogram of CA I. This single mechanical system with four-way cross could carry out CIEF-ESI-MS without washing the line, discontinuity of electric circuit and reinstallation of ESI tip. Coupling FT/MS with CIEF shows the isotopic mass distribution as well as the charge distribution of CA I in the mass spectrum. With further optimization of the operational parameters of the new interface, this online CIEF-ESI-MS can be performed for the complex protein samples by achieving protein separation according to their $p \mathrm{I}$ values.

\section{Acknowledgement}

This article is dedicated to Dr. Hun-Young So on the occasion of his honorable retirement after his life-long contribution to chemistry in Korea Research Institute of Standards and Science.

\section{References}

1. Righetti, P. G. Isoelectric Focusing: Theory, Methodology and Applications; Elsevier Biomedical Press: Amsterdam, 1983.

2. Hjerten, S.; Zhu, M. D. Journal of Chromatogr. 1985, 346, 265.

3. Tang, Q.; Harrata, A. K.; Lee, C. S. Anal. Chem. 1995, $67,3515$.

4. Tang, Q.; Harrata, A. K.; Lee, C. S. Anal. Chem. 1997, $69,3177$.

5. Martinovic, S.; Berger, S. J.; Pasa-Polic, L.; Smith, R. D. Anal. Chem. 2000, 72, 5356.

6. Clarke, N. J.; Naylor, S. Biomed. Chromatogr. 2002, 16, 287.

7. Kuroda, Y.; Yukinaga, H.; Kitano, M.; Noguchi, T.; Nemati, M.; Shibukawa, A.; Nakagawa, T.; Matsuzaki, K. J. Pharm. Biomed. Anal. 2005, 37, 423.

8. Mokaddem, M.; Gareil, P.; Varenne, A. Electrophoresis 2009, 30, 4040 .

9. Smith, R. D.; Olivares, J. A.; Nguyen, N. T.; Udseth, H. R. Anal. Chem. 1988, 60, 436.

10. Pleasance, S.; Thibault, P.; Kelly, J. J. Chromatogr. 1992, 591, 325.

11. Shen, Y.; Smith, R. D. J. Microcol. Separations 2000, 12, 135. 